\title{
Pubic osteomyelitis presenting as irritable hip
}

\author{
J.R. Weinberg, ${ }^{1 *}$ L. Berman, ${ }^{2}$ G. Dootson ${ }^{1} \dagger$ and R. Mitchell ${ }^{2}$ \\ ${ }^{1}$ Lister Unit and ${ }^{2}$ Department of Radiology, Northwick Park Hospital and Clinical Research Centre, Watford \\ Road, Harrow, Middlesex
}

\begin{abstract}
Summary: Two cases of pubic osteomyelitis presenting as a painful hip are reported. In both cases the diagnosis was delayed by the unusual presentation and by the limited radiological investigation.

Pubic osteomyelitis is rare in childhood but should be considered in the differential diagnosis of the 'irritable hip'.
\end{abstract}

\section{Introduction}

This paper reports two children with pubic osteomyelitis presenting as cases of 'irritable hip'.

\section{Case reports}

Case 1

An eight year old girl presented with a four week history of pain in the left buttock and hip which persisted at rest and was accompanied by a low grade pyrexia. Her symptoms resolved and the temperature settled after treatment with aspirin from her general practitioner. Two weeks later she developed bilateral hip pain and experienced difficulty in rising from a chair.

On admission she was afebrile. There was a full range of movement in both hips with pain at the limit of extreme adduction on the left. There was tenderness over the left adductor tendon.

Blood tests were normal apart from an erythrocyte sedimentation rate (ESR) of $30 \mathrm{~mm} / \mathrm{h}$. An initial pelvic radiograph was considered normal. Ultrasound of the hip excluded an effusion. Subsequent radionuclide bone scanning showed increased uptake in the left pubic body; by this time a repeat plain radiograph was obviously abnormal. Surgical exploration revealed an abscess cavity from which Staphylococcus aureus was isolated. Recovery was uneventful on antibiotic (flucloxacillin and fusidic acid) chemotherapy.

\footnotetext{
*Correspondence and present address: J.R. Weinberg, M.R.C.P., Dept. of Medicine, Charing Cross Hospital, Fulham Palace Road, London W6, UK.

†Present address: Royal Free Hospital, Pond St., London NW3, UK.

Accepted: 29 October 1986
}

Case 2

A fifteen year old boy presented with a ten day history of vomiting, headache, sore throat and fever, followed several days later by discomfort in the right thigh. He completed a five day course of amoxycillin prescribed by his general practitioner. Two days before admission he developed pain in the right hip with difficulty in walking. There was suprapubic discomfort on micturition but no haematuria or urethral discharge.

On examination he was flushed and unwell and there was a pyrexia of $40^{\circ} \mathrm{C}$. His right hip was held in flexion, there was a full range of passive movements except for painful limitation of extreme abduction. Rectal and abdominal examination were normal. Investigation revealed a leucocytosis of $30 \times 10^{9} / 1$ ( $90 \%$ neutrophils); the ESR was $29 \mathrm{~mm} / \mathrm{h}$. The urine was normal. The initial abdominal radiograph was normal. Ultrasound excluded a hip effusion but demonstrated a pelvic mass indenting the right wall of the bladder. This was thought to be a caecal mass or appendix abscess. Computed tomographic (CT) scanning demonstrated a retropubic soft-tissue mass with adjacent bony destruction. A radiograph of the pelvis confirmed the pubic involvement, this region having been excluded from the original abdominal radiograph. An isotope bone scan demonstrated a corresponding area of increased activity and confirmed that this was a solitary lesion. Five ml of pus were aspirated, which was sterile, probably because the patient had already been given antibiotics. The patient was initially treated with flucloxacillin and gentamicin. Metronidazole was added on the basis of this being an appendix abscess. He made an uneventful recovery.

C The Fellowship of Postgraduate Medicine, 1987 


\section{Discussion}

In children osteomyelitis most commonly affects the metaphyses of the long bones, but occasionally the spinal column is affected. Haematogenous osteomyelitis of the pubis is rare and a review of the world literature ${ }^{1}$ yielded a total of 49 cases of pubic osteomyelitis in both adults and children. There have been several case reports since $e^{2,3,4,5}$ and in a survey of osteomyelitis in childhood the pelvis was involved in 4 cases out of $328 .^{6}$

Common features of the reported cases have been presentation as an 'irritable hip' and a delay in making the diagnosis. Although clinical examination of the hip is frequently normal there may be pain on active but not on passive movement. Dysuria was a feature of one of our cases and has been reported previously. ${ }^{4}$

In one of our cases and in many of the reported cases exclusion of the pubis from the radiograph resulted in diagnostic delay. The need for an initial full pelvic radiograph without gonadal shielding in children with hip pain should be emphasized.

Although many of the reported cases were subjected to aspiration of the hip we consider this unnecessary in the absence of ultrasound evidence of an effusion. The technique is well described. ${ }^{7}$ In the presence of acute hip symptoms a normal hip ultrasound scan is a strong

\section{References}

1. Heldrich, F. \& Harris, V. Osteomyelitis of the pubis. Acta Paediatr Scand 1979, 68: 39-41.

2. Greenstone, G. \& Greensides, R. Osteomyelitis of the pelvis. A diagnostic problem. Am J Dis Child 1978, 132: 581-582.

3. Edwards, M.S., Baker, C.J., Granberry, M. \& Barret, F.F. Pelvic osteomyelitis in children. Pediatrics 1978, 61: 62-67.

4. Simms, R.G., St John Brown, B., Hyndman, J.L. \& Goldbloom, R.B. Osteomyelitis of the pelvis in childhood. Can Med Assoc J 1981, 124: 1028-1030.

5. Highland, T.R. \& Lamont, R.L. Osteomyelitis of the pelvis in children. J Bone Joint Surg 1983, 65A: 230-234.

6. Gilmour, W.N. Acute haematogenous osteomyelitis. $J$ indication that the cause lies elsewhere. While an isotope bone scan may be helpful and in fact suggested the diagnosis in one of our patients, bladder activity overlying the pubis may be misleading. Early post micturition or oblique views could overcome this. A case of pubic osteomyelitis producing an area of reduced activity on bone scan has been reported ${ }^{8}$ and it is conceivable that this may lead to diagnostic confusion.

Most cases of haematogenous osteomyelitis are caused by Staphylococcus aureus. Therefore therapy should be directed at this organism. Urgent explora- $\vec{\circ}$ tion to relieve sub-periosteal pus and obtain material for bacteriological investigation is important. This can either be at open biopsy or by needle aspiration under radiological control. Occasionally species such as Haemophilus may be found. ${ }^{9}$

Treatment should be prolonged and 4 weeks of intravenous therapy has been suggested; ${ }^{10}$ many physicians would then continue with oral antibiotics for several more weeks.

\section{Acknowledgements}

We would like to thank Dr B. Ansell and Dr H. Smith for permission to report patients under their care.

Bone Joint Surg 1962, 44B: 841-853.

7. Wilson, D.J., Green, D.J. \& MacLarnon, J.L. Arthrosonography of the painful hip. Clin Radiol 1984, 35: 17-19.

8. Trackler, R.T. Miller, K.E., Sutherland, D.H. \& Chadwick, D.L. Childhood pelvic osteomyelitis presenting as a "cold" lesion on bone scan: Case report. J Nucl Med 1976, 17: 620-622.

9. Learmonth, I.D., Dall, G. \& Pollock, D.J. Acute osteomyelitis and septic arthritis in children. A simple $\dot{\sigma}$ approach to treatment. $S$ Afr Med J 1984, 65: 117-120.

10. Norden, C.W. In: Mandell, G.L., Douglas, R., Bennett, J.G. (eds) Principles and Practice of Infectious Disease. J Wiley \& Sons, New York, 1979, pp 954-955. 